\title{
Washer Device
}

National Cancer Institute

\section{Source}

National Cancer Institute. Washer Device. NCI Thesaurus. Code C50267.

A device designed to cleanse an object, or a flattened disk used as a mechanical seal between objects. 